\title{
Ameliorative Efficacy of Ethanolic Extracts of Curcuma longa (turmeric) Roots and Cassia occidentalis Leaves on Potassium Induced Kidney Damage in Albino Rats
}

\author{
Nnama T.N, Anibueze C.I.P, Okwara B.O, Okafor M.C \\ *Abia State University, Uturu Abia State \\ *Enugu State University of Science and Technology \\ *University of Nigeria Teaching Hospital.
}

\begin{abstract}
The emphasis of harmful health challenges caused by preserved food or processed food is a global problem and the need to reduce its effect on the vital organs in the body has been the subject of great concern to researchers. The present study seeks to evaluate the efficacy of ethanolic extracts of Curcuma longa (turmeric) roots and Cassia occidentalis leaves on potassium induced kidney damage in albino rats. Fifty adult male rats weighing about $100 \mathrm{~g}-200 \mathrm{~g}$ classified into ten groups (I-X) were used in the study. Group1 served as control and were administered only with distilled water and rat feeds ad libitum all throughout the experiment. Group II served as negative (-ve) control and were administered $50 \mathrm{mg} / \mathrm{kg}$ bodyweight of potassium bromate orally. Groups III, IV, VII and VIII received $50 \mathrm{mg} / \mathrm{kg}$ bodyweight of potassium bromate for two weeks thereafter received $50 \mathrm{mg} / \mathrm{kg}$ bodyweight with $500 \mathrm{mg}$ and $1000 \mathrm{mg} / \mathrm{kg}$ body weight of ethanolic root extract of Curcuma longa and leaves extract Cassia occidentalis respectively for two weeks. Groups V, VI, and IX and $X$ received $500 \mathrm{mg}$ and $1000 \mathrm{mg} / \mathrm{kg}$ bodyweight of ethanolic root extract of Curcuma longa and leaves extract Cassia occidentalis for two weeks thereafter received $50 \mathrm{mg} / \mathrm{kg}$ bodyweight of potassium bromate withethanolic root extract of Curcuma longa and leaves extract Cassia occidentalis respectively for two weeks. The rats at the end of 28 days were anaesthetised, blood samples were collected and the kidneys were harvested. The result of biochemical analysis revealed significant decrease in the level of biochemical parameters following administration of $500 \mathrm{mg} / \mathrm{kg}$ and $1000 \mathrm{mg} / \mathrm{kg}$ body weight of Curcuma longa and Cassia occidentalis ethanolic leaf extract for curative and protective purpose when compared with group II (+ve control) that received $50 \mathrm{mg} / \mathrm{kg}$ body weight of potassium bromate. Histological findings revealed restoration and protection of the extracts on the kidney architecture of male albino rats. Results obtained thus showed that oral administration of ethanolic root extracts of Curcuma longa and leaf extract of Cassia occidentalis may possess preventive and therapeutic purpose against kidney damage.
\end{abstract}

Keywords:- Cassia Occidentalis, Curcuma Longa, Kidney, Potassium Bromate.

\section{INTRODUCTION}

The levels of food contamination have reached an allnew level. To preserve the taste, freshness, and colour of the foods, even fresh fruits and vegetables are loaded with chemicals and preservatives (www.kent.co.in, 2019). Taking into consideration the increased use of chemicals and preservatives, junk food may be labelled to be dangerous and its consumption will become a matter of choice. However, toxic compound may be formed through metabolism of non-toxic additives either during food processing or after ingestion. Potassium bromate $\left(\mathrm{KBrO}_{3}\right)$ is an oxidizing agent that is commonly used in cosmetic products (such as permanent hair weaving solutions and dying of textiles), bread and cake improvers, preservatives of packed foods, a food additive, and is a major tap water pollutant (Kakehashi et al., 2013). Potassium bromate is very stable in the body and only small amount reduced to bromide by glutathione processes in the liver and kidney (Kutom et al., 1990). Potassium bromate is excreted in urine either as bromate or bromide (Fujieet al., 1984). In several countries, including the United States, it is still used (legally and illegally) as a bread and cake improver even though it has been associated with the development of several organ damage (Oloyede and Sunmonu, 2009; Kakehashi et al., 2013).

Natural medicinal products are increasingly gaining popularity and used worldwide as complementary alternative therapies (WHO, 2003), due to their abundance in nature with an estimated record of approximately 106263 potentially beneficial substances (Drew, 2000). Among such therapeutic preparations are plant-derived phytomedicines, nutraceuticals and cosmeceuticals (Drew, 2000). Many medicinal plants have been reported to possess hepato- and reno-protective effects; such plants include the like of Cassia occidentalis (Al-Snafi, 2015) and Curcuma longa (turmeric) (Mahdi et al., 2019).Curcumalonga (Turmeric) is aperennial herbaceous plant of the ginger family (Zingiberaceae), distributed mainly throughout tropical and subtropical regions of the world (Joe et al., 2004). Its tuberous rhizomes, or underground stems, have been used from antiquity as a condiment, a textile dye, and medically as an aromatic stimulant (Joe et al., 2004).Curcumin 
(diferuloylmethane) is a yellow colouring ingredient of the spice Turmeric obtained from the rhizome of Curcuma longa Linn (Zingiberaceae) (Joe et al., 2004). Curcuma longa possesses antioxidant (Maizura et al., 2011), antitumor (Kunnumakkara et al., 2007), antimicrobial (Kim et al., 2005), anti-inflammatory (Kohli et al., 2005), wound healing (Panchatcharam et al., 2006), and gastro-protective activities (Miriyala et al., 2007) commonly known as Kunyit in Malaysia and turmeric in Nigeria, it is a popular ingredient for preparing culinary dishes (Phansawan and Poungbangpho, 2007). Curcuma longa has been documented to have Reno and hepato- protective potentials against toxicity induced by rifampicin and isoniazid in rats (Mahdi et al., 2019). In folk medicine, the rhizome juice from Curcuma longa has also been used in the treatment of many diseases such as anthelmintic, asthma, gonorrhea and urinary tract infections (Phansawan andPoungbangpho, 2007). While Cassia occidentalis commonly called coffee senna (Haselwood and Motter, 1966) is locally known as stinking weed (Henty et al., 1975). It has a single purplish stem and sparse branching (Long and Lakela, 1976). The paste of the leaf is externally applied on healing wounds, sores, itch, skin diseases, bone fracture, ringworm and throat infection (Yadav et al., 2009). Other uses of this plant include as diuretics, laxative, anti-bacteria, antiinflammatory, hepato-protective and anti-fungal (Yadav et al., 2009). Extracts from the plant leaves were repeatedly used folklorically as an analgesic, antibacterial, antifungal, anti-inflammatory, antiseptic, antispasmodic, anti-parasitic, antiviral, carminative, diaphroretic, emmanagogue, febrifuge, insecticidal, immune-stimulant, laxative, purgative, sudorific, hepatoprotective effect (Nwaehujor et al.,2011) and vermifuge (Gaind et al., 1966).Histopathological observations have also shown the hepato-protectivity of the root sample (Usha et al., 2007). However, ingestion of large amounts of coffee senna seeds caused deaths of cows, goats, horses and pigs (Timm and Riet-Correa, 1997). However, the study is aimed to evaluate the efficacy of ethanolic extracts of Curcuma longa (turmeric) roots and Cassia occidentalis leaves on potassium induced kidney damage in albino rats.

\section{MATERIALS AND METHODS}

\section{- Location and duration of experiment}

This study was conducted in the Department of Anatomy, Faculty of Basic Medical Sciences Abia State University, Uturu Nigeria. The experimental Animals were housed at the Animal House of Faculty of Basic Medical Sciences Abia State University, Uturu, Nigeria. The animals were acclimatized for two weeks before the administration of extracts that lasted for 28days.

\section{- Ethical approval}

Ethical approval following international standard on Ethical Guidelines for the Use of Animals in Research (1999) was sort and obtained from the Faculty of Basic Medical Sciences Ethics Committee, Abia State University, Uturu Nigeria.

\section{- Plant source and identification}

Matured roots $(5 \mathrm{~kg})$ of Curcuma longa and leaves ofCassia occidentalis ( $2 \mathrm{~kg}$ ) were obtained and procured from Nkwo Nnewi Market at Nnewi, in Nnewi-North Local Government Area of Anambra State, Nigeria. The Botanical Identification of the plant was done by $\mathrm{Mr}$. Egboka Tochukwu (a Botanist) of the Department of Botany, Nnamdi Azikiwe University Awka, Anambra State.

\section{- Procurement of potassium bromate}

Potassium bromate (Sigma Aldrich, Germany) was procured from a certified pharmaceutical shop at Onitsha Market Anambra state, Nigeria.

\section{- Preparation of plant materials and extraction of plant materials}

The fresh leaves of Cassia occidentalis and roots of Curcuma longa were washed with clean water to remove dirt and sand. They were afterwards separated, drained and chopped into very little pieces; shades dried and then pulverize into fine powder. Five hundred grams $(500 \mathrm{~g})$ of the powdered form of the leaves of Cassia occidentalis and root of Curcumalonga were separately macerated in 1.5 litres of ethanol for 48 hours. The solution was afterwards filtered with whatman no 4 filter paper and the filtrate concentrated to a semi-solid residue in an oven at $60^{\circ \mathrm{C}}$. The semi-solid extract obtained was the stored in a refrigerator, at a temperature of $7^{0 \mathrm{C}}$.

\section{- Acute toxicity ( $\left.L D_{50}\right)$ (median lethal dose) of Curcuma longa}

The acute toxicity study of the Curcuma longa root extract, ethanolic leaf extract of Cassia occidentalis and potassium bromatewas determined using modified Lorke's (1983) method.

$\mathrm{LD}_{50}$ of ethanolic leaf extract of Curcuma longa and ethanolic leaf extract of Cassia occidentalis is above $5000 \mathrm{mg} / \mathrm{kg}$ while the LD50of potassium bromate in this research via oral route was found to be $316.23 \mathrm{mg} / \mathrm{kg}$.

\section{Experimental Design}

- Animal care

All experimental investigations were done in compliance with "humane animal" as stated in the "Guide to the care and use of Laboratory Animals Resources" (NRC, 2011).

\section{- Conditioning animals}

A total of 50 male albino rats with weigh range of 100-200g were used for this study. Animals were acclimatized for two (2) weeks in the animal house of the Faculty of Basic Medical Sciences, Abia State University, Uturu. The animals were maintained under standard and good laboratory conditions of light (12hours), temperature $\left(23 \pm 2^{\circ} \mathrm{c}\right)$, humidity $(60 \%-70 \%)$ and ventilation. They were given standard rat diet (growers mesh rat pellets, Grand Cereals Ltd Enugu) purchased from the same farm to avoid 
changes in dietary compositions and weight variability and adequate water ad libitum would be given.

\section{- Preparation of the extract for administration}

The extract was prepared on daily basis by dissolving $1 \mathrm{~g}$ of the extracts in $10 \mathrm{mls}$ of distilled water (Stock solution). Potassium bromate and Curcumalonga ethanolicroot extract and Cassia occidentalis ethanolicleaf extractwere administered orally using 2 mls syringe and a cannula.

\section{Animal Grouping and Experimental Protocol}

\section{- Animal grouping}

After acclimatization, animals were divided into ten groups ( $\mathrm{I}-\mathrm{X})$ of five animal each $(\mathrm{N}=5)$ and were dosed accordingly, noting the result from the acute toxicity test (Lethal dose Ld50) of Curcuma longa, Cassia occidentalis and potassium bromate; I, to $X(n=5)$. Group 1 , was the control group with animals receiving feeds (growers mesh rat pellets, Grand Cereals Ltd Enugu) and distilled water only. Group II was the positive control (administered potassium bromate), III, to $\mathrm{X}$ were the treated group and treated as follows;

$\checkmark$ Group III received $50 \mathrm{mg} / \mathrm{kg}$ body weight of potassium bromate daily for 2 weeks, thereafter; received $50 \mathrm{mg} / \mathrm{kg}$ body weight of potassium bromate and Curcuma longa ethanolic root extract $500 \mathrm{mg} / \mathrm{kg}$ body weight for 2weeks.

$\checkmark$ Group IV received $50 \mathrm{mg} / \mathrm{kg}$ body weight of potassium bromate daily for 2 weeks thereafter; received $50 \mathrm{mg} / \mathrm{kg}$ body weight of potassium bromate and Curcuma longa ethanolic root extract $1000 \mathrm{mg} / \mathrm{kg}$ body weight daily for 2 weeks.

$\checkmark$ Group V received Curcuma longa ethanolic root extract $500 \mathrm{mg} / \mathrm{kg}$ body weight for 2 weeks, thereafter; received $50 \mathrm{mg} / \mathrm{kg}$ body weight of potassium bromate and Curcuma longa ethanolic root extract $500 \mathrm{mg} / \mathrm{kg}$ body weight for 2 weeks.

$\checkmark$ Group VI received Curcuma longa ethanolic root extract $1000 \mathrm{mg} / \mathrm{kg}$ body weight for 2 weeks, thereafter; received $50 \mathrm{mg} / \mathrm{kg}$ body weight of potassium bromate and Curcuma longa ethanolic root extract $1000 \mathrm{mg} / \mathrm{kg}$ for 2 weeks.

$\checkmark$ Group VII received $50 \mathrm{mg} / \mathrm{kg}$ body weight of potassium bromate daily for 2 weeks thereafter; received $50 \mathrm{mg} / \mathrm{kg}$ body weight of potassium bromate Cassia occidentalis ethanolicleaf extract $500 \mathrm{mg} / \mathrm{kg}$ body weight for 2 weeks.

$\checkmark$ Group VIII received $50 \mathrm{mg} / \mathrm{kg}$ body weight of potassium bromate daily for 2 weeks, thereafter; received $50 \mathrm{mg} / \mathrm{kg}$ body weight of potassium bromate $/ \mathrm{kg}$ and Cassia occidentalis ethanolic leaf extract $1000 \mathrm{mg} / \mathrm{kg}$ body weight for 2 weeks.

$\checkmark$ Group IX received Cassia occidentalis ethanolic leaf extract $500 \mathrm{mg} / \mathrm{kg}$ body weight for 2 weeks thereafter; received $50 \mathrm{mg} / \mathrm{kg}$ body weight of potassium bromate and Cassia occidentalis leafextract $500 \mathrm{mg} / \mathrm{kg}$ body weight for 2 weeks. $\checkmark$ Group X received Cassia occidentalis ethanolic leaf extract $1000 \mathrm{mg} / \mathrm{kg}$ body weight for 2 weeks, thereafter; received $50 \mathrm{mg} / \mathrm{kg}$ body weight of potassium bromate and Cassia occidentalis leafextract $1000 \mathrm{mg} / \mathrm{kg}$ body weight for 2 weeks.

- Observation of behavioural changes in the animals

Visual observations for mortality, behavioural pattern changes such as weakness, aggressiveness, food or water refusal, diarrhoea, salivation, discharge from eyes and ears, noisy breathing, changes in locomotor activity, injury, pain or any signs of illness in each treated group were monitored and documented carefully on daily basis throughout the experiment period.

\section{- Organ Harvest and Collection of Blood Samples (Necropsy)}

Twenty-four hours after the last substrate administration, the rats were painlesslyanesthetized with chloroform using chloroform in a closed jar. Blood samples were collected directly from the heart (via cardiac puncture) using $5 \mathrm{ml}$ syringes. Blood samples collected where placed in specific sterilized plastic containers required for each test procedure. Blood samples were taken and allowed to clot at room temperature for 30minutes then centrifuged (Hittich EBA35) at 3000 r.p.m. Sera were separated and stored at $20^{\circ} \mathrm{C}$ until analyzed for biochemical parameters; such as: urea, creatinine and electrolytes (sodium, potassium, chloride and bicarbonate ions) of the kidney. Thereafter the animals were sacrificed by cervical dislocation. Their livers and kidneys collected after which the remains of the animals were properly buried.

\section{- Organ harvest}

This procedure was carried out by positioning the sacrificed animals in a supine position on a dissecting board with their ventral side facing upwards and the four limbs stretched and pinned to the dissecting board for easy dissection. A sharp surgical blade fixed on a blade holder was used to make a gentle midline incision along abdomino-pelvic region on each rat to avoid damage to the visceral organs. Another incision was made horizontally along the upper part of the pelvis by using a pair of dissecting forceps and scissors, the skin was reflected, the superficial and deep fascia, the sternum was carefully dissected to expose the thoracic cavity and further down the abdomino-pelvic cavity was exposed. The kidneys were traced, harvested and examined macroscopically for any lesions or abnormalities. The weight of the organs from all the groups would be measured and recorded. The harvested organs were placed in normal saline to maintain normal physiological conditions after which they were fixed in $10 \%$ formalin. The relative organ weight of each animal would be calculated using Sahgal et al., 2010 method as follows.

Relative organ weight: absolute organ weight body weight of rat on the day of sacrifice 
All of the individual organs were observed macroscopically and their appearance was compared between both treated and control groups. The fixed organs were further processed for histological observations.

\section{- Statistical data analysis}

Data of weekly Animal body weights, kidney weights (relative) and biochemical parameters were analysed using the statistical package of social sciences (SPSS) software version 21.0 (SPSS) Inc. Chicago and Microsoft. Statistical analysis of variance was carried out using student T-test and one way ANOVA (SPSS 21.0). Values obtained were recorded in mean+ standard deviation. A value of $p<0.05$ would be used as the level of significance.

\section{RESULTS}

Observations on body weight for curative groups

\begin{tabular}{|c|c|c|c|c|c|c|}
\hline & & MEAN & \pm SEM & WD & P-value & T-Value \\
\hline \multirow[t]{2}{*}{ Group I (Control) } & Initial weight $(\mathrm{g})$ & 121.00 & \pm 6.21 & 35.40 & $0.033 *$ & -3.19 \\
\hline & Final weight (g) & 156.40 & \pm 5.85 & & & \\
\hline \multirow{2}{*}{$\begin{array}{c}\text { Group II (Potassium Bromate } \\
\text { Only) }\end{array}$} & Initial weight (g) & 126.40 & \pm 3.72 & -4.00 & 0.477 & 0.84 \\
\hline & Final weight (g) & 122.40 & \pm 2.65 & & & \\
\hline \multirow{2}{*}{$\begin{array}{c}\text { Group III }(50 \mathrm{mg} / \mathrm{kg} \text { of } \mathrm{KBrO} 3+ \\
500 \mathrm{mg} / \mathrm{kg} \text { of C. Longa })\end{array}$} & Initial weight (g) & 114.20 & \pm 4.67 & 14.80 & 0.066 & -2.51 \\
\hline & Final weight (g) & 129.00 & \pm 3.57 & & & \\
\hline \multirow{2}{*}{$\begin{array}{c}\text { Group IV }(50 \mathrm{mg} / \mathrm{kg} \text { of } \mathrm{KBrO} 3+ \\
1000 \mathrm{mg} / \mathrm{kg} \text { of C. Longa })\end{array}$} & Initial weight $(\mathrm{g})$ & 116.80 & \pm 3.73 & 13.00 & $0.016 *$ & -3.99 \\
\hline & Final weight $(\mathrm{g})$ & 129.80 & \pm 3.95 & & & \\
\hline \multirow{2}{*}{$\begin{array}{c}\text { Group VII }(50 \mathrm{mg} / \mathrm{kg} \text { of } \mathrm{KBrO} 3+ \\
500 \mathrm{mg} / \mathrm{kg} \text { of C. Occidentalis })\end{array}$} & Initial weight $(\mathrm{g})$ & 116.60 & \pm 3.02 & 10.00 & $0.003^{*}$ & -6.74 \\
\hline & Final weight (g) & 126.60 & \pm 1.86 & & & \\
\hline \multirow{2}{*}{\begin{tabular}{|c|} 
Group VIII $(50 \mathrm{mg} / \mathrm{kg}$ of $\mathrm{KBrO} 3+$ \\
$1000 \mathrm{mg} / \mathrm{kg}$ of C. Occidentalis $)$
\end{tabular}} & Initial weight $(\mathrm{g})$ & 114.20 & \pm 2.20 & 16.40 & $0.008^{*}$ & -4.86 \\
\hline & Final weight $(\mathrm{g})$ & 130.60 & \pm 2.24 & & & \\
\hline
\end{tabular}

Table 1:- shows the comparative effect of C. longa and C. occidentalis on Potassium bromate induced toxicity on body weight for curative groups

Data was analyzed using t-test and values were considered significant at $p<0.05$. $* \mathrm{P}<0.05$ means significant. WD=weight difference.

Observations on body weight for protective groups

\begin{tabular}{|c|c|c|c|c|c|c|}
\hline & & MEAN & \pm SEM & WD & P-value & T-Value \\
\hline \multirow[t]{2}{*}{ Group I (Control) } & Initial weight (g) & 121.00 & \pm 6.21 & 35.40 & $0.033^{*}$ & -3.19 \\
\hline & Final weight (g) & 156.40 & \pm 5.85 & & & \\
\hline \multirow{2}{*}{$\begin{array}{c}\text { Group II (Potassium Bromate } \\
\text { Only) }\end{array}$} & Initial weight (g) & 126.40 & \pm 3.72 & -4.00 & 0.477 & 0.84 \\
\hline & Final weight (g) & 122.40 & \pm 2.65 & & & \\
\hline \multirow{2}{*}{$\begin{array}{c}\text { Group V (500mg/kg of C. } \\
\text { Longa + 50mg/kg of KBrO3) }\end{array}$} & Initial weight (g) & 107.60 & \pm 1.77 & 25.80 & $0.001 *$ & -9.52 \\
\hline & Final weight (g) & 133.40 & \pm 1.86 & & & \\
\hline \multirow{2}{*}{$\begin{array}{l}\text { Group VI }(1000 \mathrm{mg} / \mathrm{kg} \text { of C. } \\
\text { Longa + 50mg/kg of KBrO3) }\end{array}$} & Initial weight (g) & 110.80 & \pm 4.35 & 23.20 & $0.008^{*}$ & -4.84 \\
\hline & Final weight (g) & 134.00 & \pm 2.58 & & & \\
\hline \multirow{2}{*}{$\begin{array}{c}\text { Group IX }(500 \mathrm{mg} / \mathrm{kg} \text { of } \mathrm{C} \text {. } \\
\text { Occidentalis }+50 \mathrm{mg} / \mathrm{kg} \text { of } \\
\text { KBrO3) }\end{array}$} & Initial weight (g) & 114.60 & \pm 5.20 & 25.20 & $0.013^{*}$ & -4.22 \\
\hline & Final weight (g) & 139.80 & \pm 4.10 & & & \\
\hline \multirow{2}{*}{$\begin{array}{c}\text { Group X (1000mg/kg of C. } \\
\text { Occidentalis }+50 \mathrm{mg} / \mathrm{kg} \text { of } \\
\text { KBrO3) }\end{array}$} & Initial weight (g) & 115.20 & \pm 4.81 & 30.40 & $0.042 *$ & -2.93 \\
\hline & Final weight (g) & 145.60 & \pm 8.03 & & & \\
\hline
\end{tabular}

Table 2:- shows the comparative effect of $C$. longa and C. occidentalis on Potassium bromate induced toxicity on body weight for protective groups

Data was analyzed using t-test and values were considered significant at $p<0.05$. $* \mathrm{P}<0.05$ means significant. WD=weight difference. 
Observations on relative kidney for curative groups

\begin{tabular}{|c|c|c|c|c|c|}
\hline & & MEAN & \pm SEM & $\mathrm{P}$-value & F-Value \\
\hline \multirow{6}{*}{$\begin{array}{l}\text { Relative kidney } \\
\text { weight (g) }\end{array}$} & Group I (Control) & 0.18 & \pm 0.00 & 0.201 & \\
\hline & Group II (Potassium Bromate Only) & 0.16 & \pm 0.00 & & \\
\hline & $\begin{array}{c}\text { Group III (50mg/kg of KBrO3 + 500mg/kg of } \\
\text { C. Longa) }\end{array}$ & 0.17 & \pm 0.02 & 0.385 & \\
\hline & $\begin{array}{c}\text { Group IV (50mg/kg of KBrO3 }+1000 \mathrm{mg} / \mathrm{kg} \text { of } \\
\text { C. Longa) }\end{array}$ & 0.21 & \pm 0.01 & $0.008 *$ & 5.06 \\
\hline & $\begin{array}{c}\text { Group VII (50mg/kg of KBrO3 + } 500 \mathrm{mg} / \mathrm{kg} \text { of } \\
\text { C. Occidentalis) }\end{array}$ & 0.20 & \pm 0.00 & $0.019 *$ & \\
\hline & $\begin{array}{c}\text { Group VIII (50mg/kg of KBrO3 }+1000 \mathrm{mg} / \mathrm{kg} \\
\text { of C. Occidentalis) }\end{array}$ & 0.22 & \pm 0.00 & $0.001 *$ & \\
\hline
\end{tabular}

Table 3:- shows the comparative effect of $C$. longa and C. occidentalis on Potassium bromate induced toxicity on relative kidney for curative groups

Data was analyzed using ANOVA followed by post Hoc Fisher's LSD Multiple Comparism, and values were considered significant at $p<0.05$.

Observations on relative kidney for protective groups

\begin{tabular}{|c|c|c|c|c|c|}
\hline & & MEAN & \pm SEM & P-value & F-Value \\
\hline \multirow{6}{*}{$\begin{array}{l}\text { Relative kidney } \\
\text { weight (g) }\end{array}$} & Group I (Control) & 0.18 & \pm 0.00 & 0.259 & \\
\hline & Group II (Potassium Bromate Only) & 0.16 & \pm 0.00 & & \\
\hline & $\begin{array}{c}\text { Group V (500mg/kg of C. Longa+50mg/kg of } \\
\text { KBrO3) }\end{array}$ & 0.20 & \pm 0.01 & 0.051 & \\
\hline & $\begin{array}{c}\text { Group VI (1000mg/kg of C. Longa+50mg/kg of } \\
\text { KBrO3) }\end{array}$ & 0.20 & \pm 0.02 & $0.035^{*}$ & 2.10 \\
\hline & $\begin{array}{c}\text { Group IX }(500 \mathrm{mg} / \mathrm{kg} \text { of C. Occidentalis + } \\
50 \mathrm{mg} / \mathrm{kg} \text { of } \mathrm{KBrO3})\end{array}$ & 0.21 & \pm 0.01 & $0.025 *$ & \\
\hline & $\begin{array}{c}\text { Group X }(1000 \mathrm{mg} / \mathrm{kg} \text { of C. Occidentalis + } \\
50 \mathrm{mg} / \mathrm{kg} \text { of } \mathrm{KBrO3})\end{array}$ & 0.21 & \pm 0.02 & $0.025 *$ & \\
\hline
\end{tabular}

Table 4:- shows the comparative effect of $C$. longa and C. occidentalis on Potassium bromate induced toxicity on relative kidney for protective groups

Data was analyzed using ANOVA followed by post Hoc Fisher's LSD Multiple Comparism, and values were considered significant at $p<0.05$. 
Observations on Urea and Creatinine concentration for curative groups

\begin{tabular}{|c|c|c|c|c|c|}
\hline & & MEAN & \pm SEM & $\mathrm{P}$-value & F-Value \\
\hline \multirow{6}{*}{$\begin{array}{c}\text { Urea Concentration } \\
(\mathrm{mg} / \mathrm{dL})\end{array}$} & Group I (Control) & 4.35 & \pm 0.08 & $0.000^{*}$ & \\
\hline & Group II (Potassium Bromate Only) & 9.60 & \pm 0.28 & & \\
\hline & \begin{tabular}{|c|} 
Group III (50mg/kg of KBrO3 + 500mg/kg \\
of C. Longa)
\end{tabular} & 4.95 & \pm 0.20 & $0.000 *$ & \\
\hline & $\begin{array}{c}\text { Group IV (50mg/kg of KBrO3 + } \\
1000 \mathrm{mg} / \mathrm{kg} \text { of C. Longa) }\end{array}$ & 4.55 & \pm 0.49 & $0.000 *$ & 52.98 \\
\hline & $\begin{array}{c}\text { Group VII (50mg/kg of KBrO3 }+500 \mathrm{mg} / \mathrm{kg} \\
\text { of C. Occidentalis) }\end{array}$ & 4.85 & \pm 0.25 & $0.000 *$ & \\
\hline & $\begin{array}{c}\text { Group VIII (50mg/kg of KBrO3 + } \\
1000 \mathrm{mg} / \mathrm{kg} \text { of C. Occidentalis) } \\
\end{array}$ & 6.05 & \pm 0.08 & $0.000 *$ & \\
\hline \multirow{6}{*}{$\begin{array}{c}\text { Creatinine } \\
\text { Concentration } \\
(\mathrm{mg} / \mathrm{dL})\end{array}$} & Group I (Control) & 52.33 & \pm 0.88 & $0.000 *$ & \\
\hline & Group II (Potassium Bromate Only) & 66.00 & \pm 0.57 & & \\
\hline & $\begin{array}{c}\text { Group III (50mg/kg of KBrO3+500mg/kg } \\
\text { of C. Longa) }\end{array}$ & 51.50 & \pm 3.75 & $0.000 *$ & 15.82 \\
\hline & $\begin{array}{c}\text { Group IV }(50 \mathrm{mg} / \mathrm{kg} \text { of } \mathrm{KBrO3}+ \\
1000 \mathrm{mg} / \mathrm{kg} \text { of C. Longa })\end{array}$ & 53.00 & \pm 0.57 & $0.000^{*}$ & \\
\hline & $\begin{array}{c}\text { Group VII (50mg/kg of KBrO3 }+500 \mathrm{mg} / \mathrm{kg} \\
\text { of C. Occidentalis) }\end{array}$ & 65.00 & \pm 0.00 & 0.675 & \\
\hline & $\begin{array}{c}\text { Group VIII (50mg/kg of KBrO3 + } \\
1000 \mathrm{mg} / \mathrm{kg} \text { of C. Occidentalis) }\end{array}$ & 59.50 & \pm 0.86 & $0.016^{*}$ & \\
\hline
\end{tabular}

Table 5:-.Shows the comparative effect of $C$. longa and C. occidentalis on Potassium bromate induced toxicity on Urea and Creatinine concentration for curative groups

Data was analyzed using ANOVA followed by post Hoc Fisher's LSD Multiple Comparism, and values were considered significant at $p<0.05$.

Observations on Urea and Creatinine concentration for protective groups

\begin{tabular}{|c|c|c|c|c|c|}
\hline & & MEAN & \pm SEM & $\mathrm{P}$-value & F-Value \\
\hline \multirow{6}{*}{$\begin{array}{c}\text { Urea } \\
\text { Concentration } \\
(\mathrm{mg} / \mathrm{dL})\end{array}$} & Group I (Control) & 4.35 & \pm 0.08 & $0.000 *$ & \\
\hline & Group II (Potassium Bromate Only) & 9.60 & \pm 0.28 & & \\
\hline & $\begin{array}{c}\text { Group V (500mg/kg of C. Longa+50mg/kg of } \\
\text { KBrO3) }\end{array}$ & 5.70 & \pm 0.29 & $0.000^{*}$ & \\
\hline & $\begin{array}{c}\text { Group VI (1000mg/kg of C. Longa+50mg/kg of } \\
\text { KBrO3) }\end{array}$ & 6.45 & \pm 1.12 & $0.001 *$ & 13.55 \\
\hline & $\begin{array}{c}\text { Group IX (500mg/kg of C. Occidentalis + } \\
50 \mathrm{mg} / \mathrm{kg} \text { of } \mathrm{KBrO3})\end{array}$ & 4.70 & \pm 0.29 & $0.000 *$ & \\
\hline & $\begin{array}{c}\text { Group X }(1000 \mathrm{mg} / \mathrm{kg} \text { of C. Occidentalis + } \\
50 \mathrm{mg} / \mathrm{kg} \text { of } \mathrm{KBrO3})\end{array}$ & 6.00 & \pm 0.17 & $0.000 *$ & \\
\hline \multirow{6}{*}{$\begin{array}{c}\text { Creatinine } \\
\text { Concentration } \\
(\mathrm{mg} / \mathrm{dL})\end{array}$} & Group I (Control) & 52.33 & \pm 0.88 & $0.000 *$ & \\
\hline & Group II (Potassium Bromate Only) & 66.00 & \pm 0.57 & & \\
\hline & $\begin{array}{c}\text { Group V (500mg/kg of C. Longa+50mg/kg of } \\
\text { KBrO3) }\end{array}$ & 52.50 & \pm 0.87 & $0.000 *$ & 34.02 \\
\hline & $\begin{array}{c}\text { Group VI (1000mg/kg of C. Longa }+50 \mathrm{mg} / \mathrm{kg} \text { of } \\
\text { KBrO3) }\end{array}$ & 48.00 & \pm 1.73 & $0.000 *$ & \\
\hline & $\begin{array}{c}\text { Group IX }(500 \mathrm{mg} / \mathrm{kg} \text { of C. Occidentalis + } \\
50 \mathrm{mg} / \mathrm{kg} \text { of } \mathrm{KBrO3})\end{array}$ & 55.00 & \pm 0.57 & $0.000 *$ & \\
\hline & $\begin{array}{c}\text { Group X }(1000 \mathrm{mg} / \mathrm{kg} \text { of C. Occidentalis + } \\
50 \mathrm{mg} / \mathrm{kg} \text { of } \mathrm{KBrO3})\end{array}$ & 43.00 & \pm 2.31 & $0.000 *$ & \\
\hline
\end{tabular}

Table 6:- Showed the comparative effect of C. longa and C. occidentalis on Potassium bromate induced toxicity on Urea and Creatinine concentration for protective groups 
Data was analyzed using ANOVA followed by post Hoc Fisher's LSD Multiple Comparism, and values were considered significant at $p<0.05$.

Observations on Sodium and Potassium ion concentration for curative groups.

\begin{tabular}{|c|c|c|c|c|c|}
\hline & & MEAN & \pm SEM & P-value & F-Value \\
\hline \multirow{6}{*}{$\begin{array}{l}\text { Sodium ion } \\
(\mathbf{m E q} / \mathbf{L})\end{array}$} & Group I (Control) & 138.00 & \pm 0.00 & $0.001 *$ & \\
\hline & Group II (Potassium Bromate Only) & 142.17 & \pm 0.44 & & \\
\hline & Group III (50mg/kg of KBrO3 + 500mg/kg of C. Longa) & 137.50 & \pm 0.86 & $0.001 *$ & \\
\hline & Group IV (50mg/kg of KBrO3 + 1000mg/kg of C. Longa) & 140.00 & \pm 0.00 & 0.052 & 5.80 \\
\hline & $\begin{array}{c}\text { Group VII (50mg/kg of KBrO3 + 500mg/kg of C. } \\
\text { Occidentalis) }\end{array}$ & 140.50 & \pm 0.86 & 0.123 & \\
\hline & $\begin{array}{l}\text { Group VIII (50mg/kg of KBrO3 }+1000 \mathrm{mg} / \mathrm{kg} \text { of C. } \\
\text { Occidentalis) }\end{array}$ & 140.00 & \pm 1.15 & 0.052 & \\
\hline \multirow{6}{*}{$\begin{array}{c}\text { Potassium } \\
\text { ion }(\mathbf{m E q} / \mathbf{L})\end{array}$} & Group I (Control) & 3.80 & \pm 0.06 & $0.002 *$ & \\
\hline & Group II (Potassium Bromate Only) & 5.35 & \pm 0.20 & & \\
\hline & Group III (50mg/kg of KBrO3 + 500mg/kg of C. Longa) & 4.20 & \pm 0.06 & $0.014 *$ & 3.79 \\
\hline & Group IV (50mg/kg of KBrO3 + 1000mg/kg of C. Longa) & 4.25 & \pm 0.60 & $0.018 *$ & \\
\hline & $\begin{array}{l}\text { Group VII (50mg/kg of KBrO3 +500mg/kg of C. } \\
\text { Occidentalis) }\end{array}$ & 4.30 & \pm 0.12 & $0.022 *$ & \\
\hline & $\begin{array}{l}\text { Group VIII (50mg/kg of KBrO3 + 1000mg/kg of C. } \\
\text { Occidentalis) }\end{array}$ & 3.90 & \pm 0.23 & $0.004 *$ & \\
\hline
\end{tabular}

Table 7:- shows the comparative effect of C. longa and C. occidentalis on Potassium bromate induced toxicity on Sodium and Potassium ion for curative groups

Data was analyzed using ANOVA followed by post Hoc Fisher's LSD Multiple Comparism, and values were considered significant at $p<0.05$.

Observations on Sodium and Potassium ion concentration for protective groups

\begin{tabular}{|c|c|c|c|c|c|}
\hline & & MEAN & $\pm \mathrm{SEM}$ & P-value & F-Value \\
\hline \multirow{6}{*}{$\begin{array}{l}\text { Sodium ion } \\
(\mathrm{mEq} / \mathrm{L})\end{array}$} & Group I (Control) & 138.00 & \pm 0.00 & $0.000^{*}$ & \\
\hline & Group II (Potassium Bromate Only) & 142.17 & \pm 0.44 & & \\
\hline & $\begin{array}{l}\text { Group V (500mg/kg of C. Longa+50mg/kg of } \\
\text { KBrO3) }\end{array}$ & 138.00 & \pm 0.00 & $0.000 *$ & \\
\hline & $\begin{array}{c}\text { Group VI (1000mg/kg of C. Longa+50mg/kg of } \\
\text { KBrO3) }\end{array}$ & 138.00 & \pm 0.57 & $0.000^{*}$ & 20.44 \\
\hline & $\begin{array}{c}\text { Group IX (500mg/kg of C. Occidentalis }+50 \mathrm{mg} / \mathrm{kg} \text { of } \\
\text { KBrO3) }\end{array}$ & 139.50 & \pm 0.28 & $0.000^{*}$ & \\
\hline & $\begin{array}{c}\text { Group X (1000mg/kg of C. Occidentalis + 50mg/kg } \\
\text { of KBrO3) }\end{array}$ & 141.00 & \pm 0.57 & 0.060 & \\
\hline \multirow{6}{*}{$\begin{array}{l}\text { Potassium ion } \\
\quad(\mathbf{m E q} / \mathbf{L})\end{array}$} & Group I (Control) & 3.80 & \pm 0.06 & $0.000^{*}$ & \\
\hline & Group II (Potassium Bromate Only) & 5.35 & \pm 0.20 & & \\
\hline & $\begin{array}{l}\text { Group V (500mg/kg of C. Longa+50mg/kg of } \\
\text { KBrO3) }\end{array}$ & 3.85 & \pm 0.49 & $0.001 *$ & 8.67 \\
\hline & $\begin{array}{c}\text { Group VI (1000mg/kg of C. Longa+50mg/kg of } \\
\text { KBrO3) }\end{array}$ & 3.70 & \pm 0.05 & $0.000 *$ & \\
\hline & $\begin{array}{c}\text { Group IX (500mg/kg of C. Occidentalis }+50 \mathrm{mg} / \mathrm{kg} \text { of } \\
\text { KBrO3) }\end{array}$ & 3.55 & \pm 0.02 & $0.000 *$ & \\
\hline & $\begin{array}{c}\text { Group X (1000mg/kg of C. Occidentalis + 50mg/kg } \\
\text { of KBrO3) }\end{array}$ & 3.75 & \pm 0.14 & $0.000 *$ & \\
\hline
\end{tabular}

Table 8:- shows the comparative effect of C. longa and C. occidentalis on Potassium bromate induced toxicity on Sodium and Potassium ion for curative groups 
Data was analyzed using ANOVA followed by post Hoc Fisher's LSD Multiple Comparism, and values were considered significant at $p<0.05$.

Observations on Chloride and Bicarbonate ionconcentration for curative groups

\begin{tabular}{|c|c|c|c|c|c|}
\hline & & MEAN & \pm SEM & P-value & F-Value \\
\hline \multirow{6}{*}{$\begin{array}{l}\text { Chloride ion } \\
(\mathbf{m E q} / \mathbf{L})\end{array}$} & Group I (Control) & 97.00 & \pm 0.57 & $0.000 *$ & \\
\hline & Group II (Potassium Bromate Only) & 103.00 & \pm 0.57 & & \\
\hline & $\begin{array}{l}\text { Group III (50mg/kg of } \mathrm{KBrO3}+500 \mathrm{mg} / \mathrm{kg} \text { of } \mathrm{C} . \\
\text { Longa) }\end{array}$ & 96.00 & \pm 0.00 & $0.000 *$ & \\
\hline & $\begin{array}{c}\text { Group IV (50mg/kg of KBrO3 + 1000mg/kg of C. } \\
\text { Longa) }\end{array}$ & 98.00 & \pm 1.15 & $0.001 *$ & 10.10 \\
\hline & $\begin{array}{c}\text { Group VII (50mg/kg of KBrO3 + 500 } \mathrm{mg} / \mathrm{kg} \text { of C. } \\
\text { Occidentalis) }\end{array}$ & 95.50 & \pm 1.44 & $0.000 *$ & \\
\hline & \begin{tabular}{|c|} 
Group VIII (50mg/kg of KBrO3 + 1000mg/kg of C. \\
Occidentalis)
\end{tabular} & 99.00 & \pm 0.57 & $0.006^{*}$ & \\
\hline \multirow{6}{*}{$\begin{array}{l}\text { Bicarbonate ion } \\
\quad(\mathrm{mEq} / \mathrm{L})\end{array}$} & Group I (Control) & 15.50 & \pm 2.59 & 0.198 & \\
\hline & Group II (Potassium Bromate Only) & 18.00 & \pm 1.15 & & \\
\hline & $\begin{array}{c}\text { Group III (50mg/kg of KBrO3 + } 500 \mathrm{mg} / \mathrm{kg} \text { of } \mathrm{C} . \\
\text { Longa) }\end{array}$ & 12.00 & \pm 1.15 & 0.080 & 4.67 \\
\hline & $\begin{array}{c}\text { Group IV (50mg/kg of KBrO3 + 1000mg/kg of C. } \\
\text { Longa) }\end{array}$ & 13.00 & \pm 0.57 & 0.198 & \\
\hline & $\begin{array}{c}\text { Group VII (50mg/kg of KBrO3 + 500 } \mathrm{Kg} / \mathrm{kg} \text { of } \mathrm{C} . \\
\text { Occidentalis) }\end{array}$ & 11.00 & \pm 0.57 & $0.030 *$ & \\
\hline & $\begin{array}{c}\text { Group VIII (50mg/kg of KBrO3 + 1000mg/kg of C. } \\
\text { Occidentalis) }\end{array}$ & 11.00 & \pm 0.00 & $0.030 *$ & \\
\hline
\end{tabular}

Table 9:- shows the comparative effect of C. longa and C. occidentalis on Potassium bromate induced toxicity on Chloride and Bicarbonate ion for curative groups

Data was analyzed using ANOVA followed by post Hoc Fisher's LSD Multiple Comparism, and values were considered significant at $p<0.05$.

Observations on Chloride and Bicarbonate ionconcentration for protective groups

\begin{tabular}{|c|c|c|c|c|c|}
\hline & & MEAN & \pm SEM & $\mathrm{P}$-value & F-Value \\
\hline \multirow{6}{*}{$\begin{array}{l}\text { Chloride ion } \\
(\mathrm{mEq} / \mathrm{L})\end{array}$} & Group I (Control) & 97.00 & \pm 0.57 & $0.000 *$ & \\
\hline & Group II (Potassium Bromate Only) & 103.00 & \pm 0.57 & & \\
\hline & Group V (500mg/kg of C. Longa+50mg/kg of KBrO3) & 96.00 & \pm 1.15 & $0.000 *$ & \\
\hline & $\begin{array}{c}\text { Group VI (1000mg/kg of C. Longa+50mg/kg of } \\
\text { KBrO3) }\end{array}$ & 98.00 & \pm 0.57 & $0.000 *$ & 15.68 \\
\hline & \begin{tabular}{|c|} 
Group IX $(500 \mathrm{mg} / \mathrm{kg}$ of C. Occidentalis $+50 \mathrm{mg} / \mathrm{kg}$ of \\
KBrO3)
\end{tabular} & 100.00 & \pm 0.00 & $0.008^{*}$ & \\
\hline & $\begin{array}{c}\text { Group X }(1000 \mathrm{mg} / \mathrm{kg} \text { of C. Occidentalis }+50 \mathrm{mg} / \mathrm{kg} \text { of } \\
\text { KBrO3) }\end{array}$ & 101.00 & \pm 0.57 & 0.055 & \\
\hline \multirow{6}{*}{$\begin{array}{c}\text { Bicarbonate ion } \\
(\mathrm{mEq} / \mathrm{L})\end{array}$} & Group I (Control) & 15.50 & \pm 2.59 & 0.202 & \\
\hline & Group II (Potassium Bromate Only) & 18.00 & \pm 1.15 & & \\
\hline & Group V (500mg/kg of C. Longa+50mg/kg of KBrO3) & 15.00 & \pm 0.57 & 0.131 & 2.97 \\
\hline & $\begin{array}{c}\text { Group VI (1000mg/kg of C. Longa+50mg/kg of } \\
\text { KBrO3) }\end{array}$ & 14.00 & \pm 1.15 & 0.052 & \\
\hline & $\begin{array}{c}\text { Group IX }(500 \mathrm{mg} / \mathrm{kg} \text { of C. Occidentalis }+50 \mathrm{mg} / \mathrm{kg} \text { of } \\
\text { KBrO3) }\end{array}$ & 12.16 & \pm 0.44 & 0.008* & \\
\hline & \begin{tabular}{|c|} 
Group X $(1000 \mathrm{mg} / \mathrm{kg}$ of C. Occidentalis $+50 \mathrm{mg} / \mathrm{kg}$ of \\
KBrO3)
\end{tabular} & 12.00 & \pm 0.57 & $0.007 *$ & \\
\hline
\end{tabular}

Table 10:- shows the comparative effect of C. longa and C. occidentalis on Potassium bromate induced toxicity on chloride and bicarbonate ion for protective groups 
Data was analyzed using ANOVA followed by post Hoc Fisher's LSD Multiple Comparism, and values were considered significant at $p<0.05$.

\section{$>$ Histopathological finding}

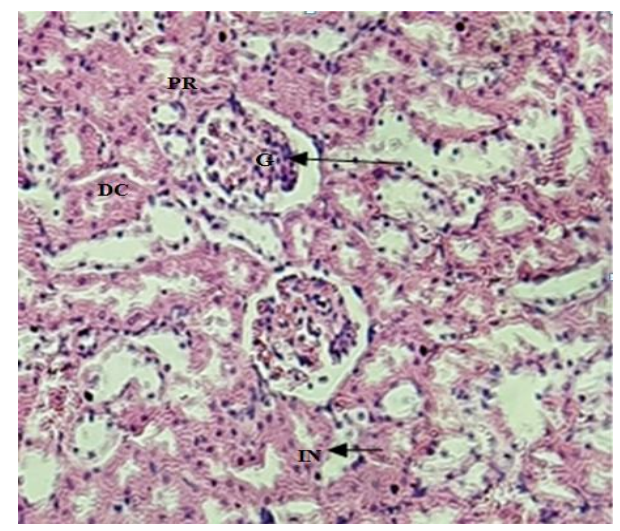

Plate1: Showed a photomicrograph of the kidney of albino rat from group I (control), the kidney histology revealed prominent renal corpuscle with glomerulus $(\mathrm{G})$ and interstitial space (IN), proximal convulated tubules (PR) and Distal convoluted tubules (DC) [H\&E×400]

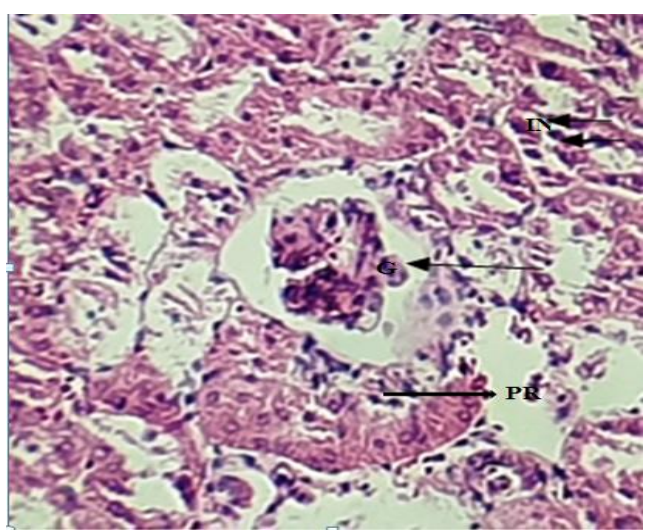

Plate 2: Showed a photomicrograph of the kidney of albino rat from group II (+ve control), the kidney histology revealed visible atrophied renal corpuscle $(\mathrm{G})$ with granulation and distorted interstitial space (IN) and tubular necrosis (PR).[H\&E×400].

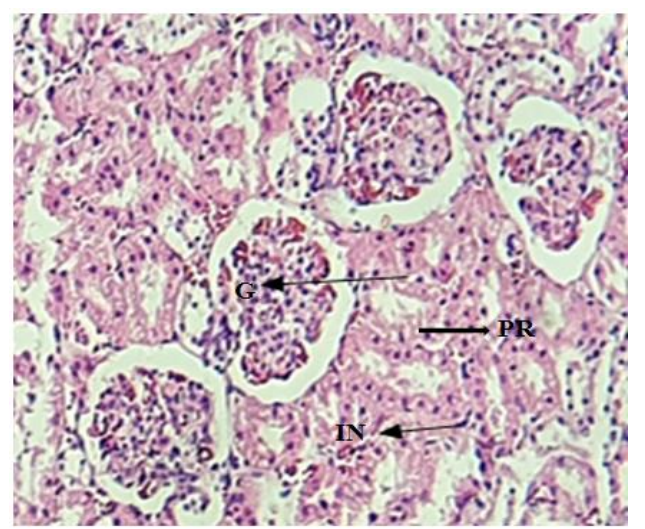

Plate 3: Showed a photomicrograph of the kidney of albino rat from group III, the kidney histology revealed prominent renal corpuscle with glomerulus $(\mathrm{G})$, mild infiltration of the

interstitial space (IN) and mild distortion of the tubules

(PR) $[\mathrm{H} \& \mathrm{E} \times 400]$.

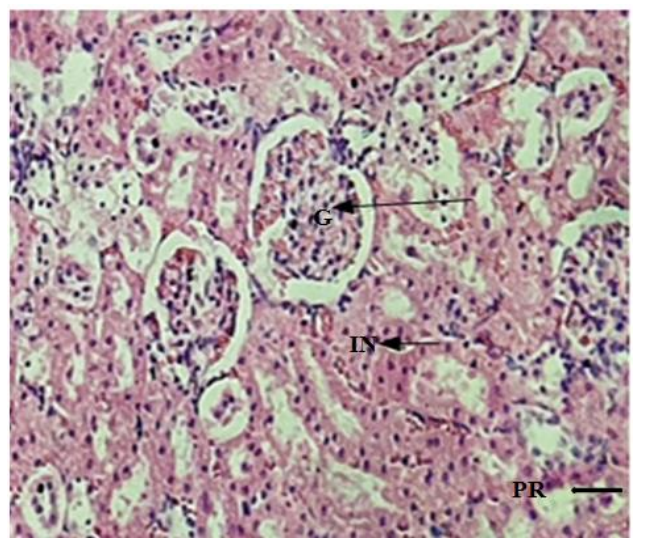

Plate 4: Showed a photomicrograph of the kidney of albino rat from group IV, the kidney histology revealed prominent renal corpuscle with glomerulus $(\mathrm{G})$ and interstitial space

(IN)with mononuclear infiltrates and normal tubules [H\&E×400].

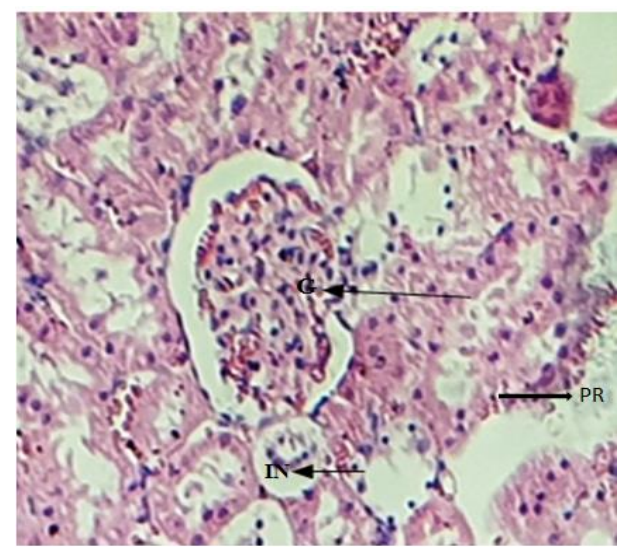

Plate 5: Showed a photomicrograph of the kidney of albino rat from group $\mathrm{V}$, the kidney histology revealed prominent renal corpuscle with glomerulus (G), interstitial space (IN) and mild tubular necrosis (PR) [H\&E×400].

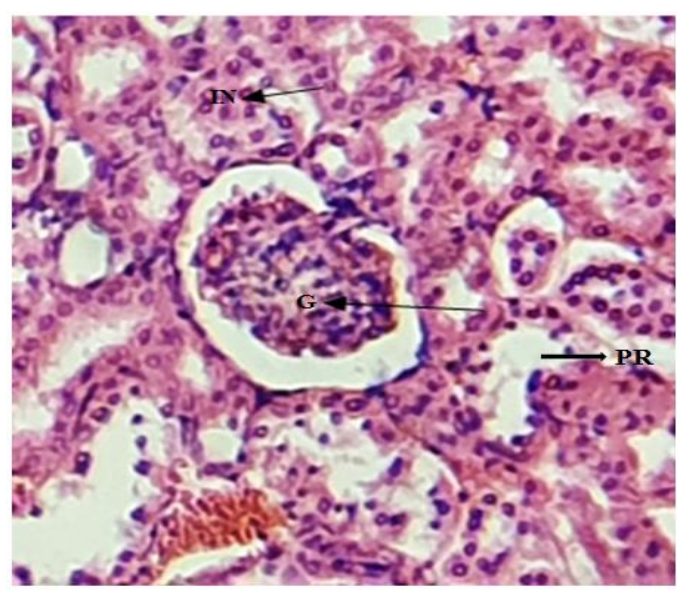

Plate 6: Showed a photomicrograph of the kidney of albino rat from group VI, the Kidney histology revealed prominent renal corpuscle with granulated glomerulus $(\mathrm{G})$ and interstitial space (IN) and mild tubular necrosis (PR) [H\&E×400]. 


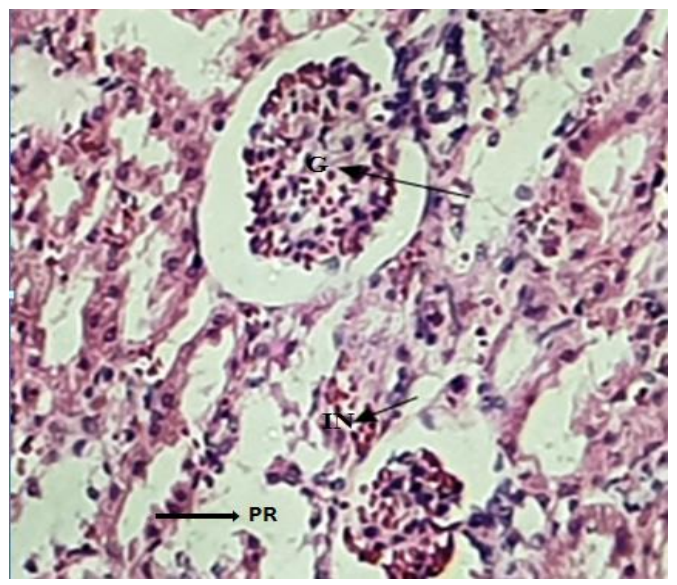

Plate 7: Showed a photomicrograph of the kidney of albino rat from group VII, the kidney histology revealed atrophied renal corpuscle with glomerulus $(\mathrm{G})$ and interstitial space

(IN) and mild tubular necrosis (PR)[H\&E×400].

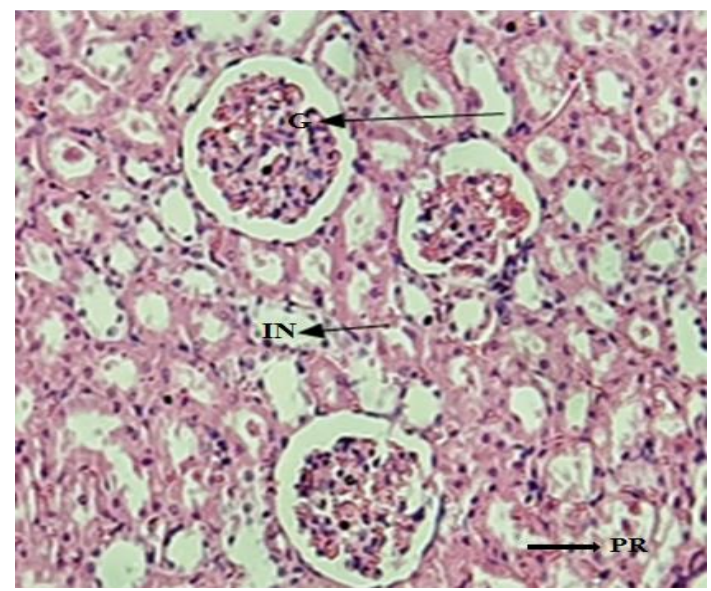

Plate 8: Showed a photomicrograph of the kidney of albino rat from group VIII, the kidney histology revealed atrophied renal corpuscle with glomerulus $(\mathrm{G})$, mild infiltration of the interstitial space (IN) and mild tubular necrosis (PR) [H\&Ex400].

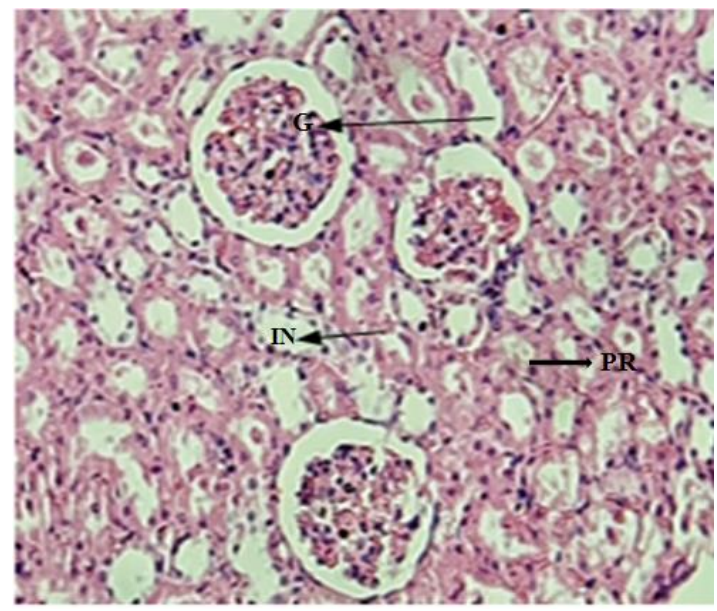

Plate 9: Showed a photomicrograph of the kidney of albino rat from group IX, the kidney histology revealed prominent renal corpuscle with glomerulus $(\mathrm{G})$ and interstitial space (IN) and tubules (PR) with no visible lesion [H\&E×400].

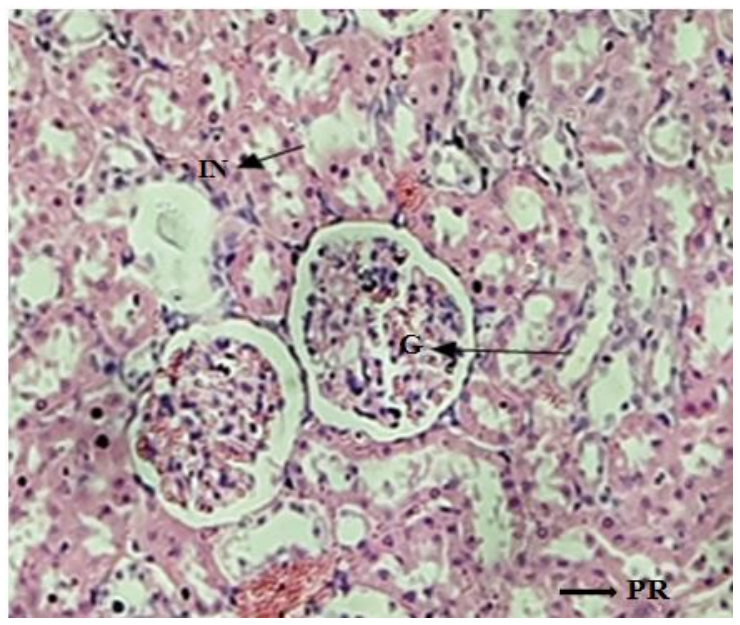

Plate 10: Showed a photomicrograph of the kidney of albino rat from group $\mathrm{X}$, the kidney histology revealed prominent renal corpuscle with glomerulus $(\mathrm{G})$ and interstitial space (IN) and normal architecture of the tubules (PR) with no visible lesion [H\&E×400].

\section{DISCUSSION}

In the present study, result obtained on curative and protective weight changes revealed a non-significant decrease in the body weight in group II that received potassium bromate only(+ve control) at $(p<0.05)$, when the initial weight was compared to the final weight of both group I (normal control) it showed significant increase, Group III and V that received $500 \mathrm{mg} / \mathrm{kg}$ body weight of Curcuma longa for curative and protective purpose had a non-significant $(p>0.05)$ increase in the body weight when the initial weight was compared to the final weight. Groups IV, VI, VII, VIII, IX and X showed significant increase in body weight when the initial weight was compared to the final weight (as seen in the table 1 and 2). The result suggests that the potassium bromate affected the body weight thereby causing decrease in the body weight and following the administration of Curcuma longa for curative and protective purpose against potassium bromate at the dose of $500 \mathrm{mg} / \mathrm{kg}$ body weight did not have significant impact in restoring the weight of the experimental rats and the effect is dose dependent (as seen in the table 1 and 2). However, at $1000 \mathrm{mg} / \mathrm{kg}$ body weight of Curcuma longa extract was significantly increased. Thus changes in body weight of rats dosed with potassium bromate only provided an imperative indication of toxicity of potassium bromate. This study agrees with that of Rehab, (2006), Farombi et al. (2000) and Watanabe et al. (2004) who reported that there were significant decrease on the body weights in rats dosed with 100 and $200 \mathrm{mg} / \mathrm{kg}$ potassium bromate in mice but in contrast with Okolie and Ikewuchi (2004) who reported a significant increase in the body weight of rats dosed $60 \mathrm{mg}$ $\mathrm{kg}^{-1}$ body wt/day of potassium bromate in rabbits.

The relative weight of the kidney in this study showed a significant $(\mathrm{p}<0.05)$ increase in the relative kidney weight in group VI, IX, and X, while there was a non-significant ( $p>0.05$ ) increase in group $\mathrm{V}$ when compared to group II; but when group I was compared to group II, there was a non-significant $(\mathrm{p}>0.05)$ decrease in the relative kidney 
weight. For curative groups a significant $(\mathrm{p}<0.05)$ increase in the relative kidney weight in group VI, IX, and X was observed, while a non-significant $(p>0.05)$ increase in group V was observed when compared to group II; but when group I was compared to group II, there was a nonsignificant $(p>0.05)$ decrease in the relative kidney weight (table 3and 4). This finding agrees with the results obtained by Farombi et al. (2000), Rehab, (2006) and Watanabe et al. (2004) who documented that administration of potassium bromate significantly decreased the relative weight of the kidney. This equally signifies curative effect and protective effect following the administration of C.occidentalis and C. longa.

The efficacy of any nephro -protective and curative drugs is dependent on its capacity of either reducing the harmful effect or restoring the normal renal physiology that has been disturbed by a nephrototoxin (Ikhajiangbe et al., 2014). In the study, the elevated level of urea and creatinine observed in group II (+ve control) that received $50 \mathrm{mg} / \mathrm{kg}$ body weight induced nephrotoxicity in the experimental rats. The administration of ethanolic root and leaves extract of Curcuma longa and Cassia occidentalis as observed in (tables 5and 6) for curative and protective properties revealed a decrease in the level of urea and creatinine in the serum of experimental groups (III to $\mathrm{X}$ ) when compared with group II (+ve control) at $\mathrm{P}<0.05$ prior to the elevation of the biochemical indices following pre and post administration of potassium bromate as nephrotoxin. The blood urea and creatinine levels increased after the kidneys were failed to remove them and other waste products from the blood (Harper, 1979). So, in this study, the elevation in blood urea and creatinine levels in potassium bromate treated rats (as seen in tables 5 and 6) is considered as suitable markers of renal dysfunction. This result is in agreement with reports of Ikhajiangbe et al., (2014), Kopple et al. (2002). Results also obtained from this current study showed that Curcuma longa and Cassia occidentalis treatment significantly attenuated the potassium bromate mediated increase in urea and creatinine levels. This effect may be related to the antioxidant properties of curcuma longa and Cassia occidentalis since it has been found that potassium bromate may be involved in the impairment of glomerular filtration rate (Pedraza et al., 2000). The protective and curative effects of Curcuma longa might also be due to ability of the extract to inhibit hydrogen peroxide-induced oxidative injury in renal cell line as has been elucidated by Cohly et al. (1998). It is thus possible to suggest that Curcuma longa is able to suppress potassium bromate nephrotoxicity in kidney as it was demonstrated in the studies with adriamycin (Venkatesan, 2000; Farombi and Ekor, 2006), and cyclosporine (Tirkey et al., 2005). On the other hand, the findings in the study agrees with findings of Isah et al., (2018), Nnama et al., (2019)and Silva et al., (2011) which revealed that Statistically, there was no significant effect seen on the renal parameters indicating oral administration of aqueous leaf extract of Senna occidentalis did not exert detrimental effect to the kidneys.
Potassium ion concentration accesses kidney function and when kidney functions detoriates the potassium levels is elevated. Sodium accesses hydration and osmotic state of the body. Chloride ions and bicarbonate ions accesses acidbase status in the electrolyte balance of humans and rats (Reyes and Gadsby, 2006). The elevation of these ions in the blood serum indicates alkalinity and the excess decrease signifies acidosis (Clement et al., 2015). In the current study, findings showed a significant $(p<0.05)$ decrease in chloride ion in groups V, VI, and IX, and a non-significant $(p>0.05)$ decrease in group $\mathrm{X}$ when compared to group II; but when group I was compared to group II, there was a significant $(p<0.05)$ increase in chloride ion while $\mathrm{Bi}$ carbonate ion result showed a significant $(p<0.05)$ decrease in group IX and X, and a non-significant $(p>0.05)$ decrease in group V and VI when compared to group II; but when group I was compared to group II, there was a nonsignificant $(p>0.05)$ increase in bicarbonate ion (table 8 and 10). it showed that kidney related diseases may be cured or protected following the administration of ethanolic root extract of Curcuma longa (Cohly et al. (1998) and ethanolic extracts of Cassia occidentalis (Isah et al., 2018, Nnama et al., 2019 and Silva et al., 2011) reduced the effect of raised electrolytes excretion by the kidney caused by administration of potassiumbromate. The result of histopathological findings of the kidney in group II (-ve control) that received $50 \mathrm{mg} / \mathrm{kg}$ body weight of potassium bromate revealed visible atrophied renal corpuscle with granulation and distorted interstitial space and tubular necrosis. The result showed that indeed that potassium bromate induced kidney damage. The result agrees with Ikhajiangbe et al., (2014) that the kidney architecture was damaged following administration of potassium bromate.

The result of histopathological findings of the kidney in group II (-ve control) that received $50 \mathrm{mg} / \mathrm{kg}$ body weight of potassium bromate revealed visible atrophied renal corpuscle with granulation and distorted interstitial space and tubular necrosis (as seen in plate 2). The result showed that indeed that potassium bromate induced kidney damage. The result agrees with Ikhajiangbe et al., (2014) that the kidney architecture was damaged following administration of potassium bromate.

The prevention and restoration of nephrotoxicity induced by potassium bromate was observed across the groups treated with Cassia occidentalis and Curcuma longa (plate 3 to 10), as both plant extracts showed curative and protective properties across the treated groups when compared with the histology of the group II (-ve control) that received $50 \mathrm{mg} / \mathrm{kg}$ body weight of potassium bromate for 4 weeks. The research agrees Hamid et al., 2014, in a study of Curcuma longa as a spice with multifunctional medicinal properties reported that the hepato-protective and reno-protective effects of Curcuma longa are mainly due to its antioxidant properties, as well as its ability to decrease the formation of pro-inflammatory cytokines (Govindarajan, 1980, Silva et al. (2011), Ammon et al., 1992, Ammon and Wahl, 1991). 


\section{CONCLUSION}

This study suggests that oral administration of ethanolic root extract of Curcuma longa and ethanolic leaves extract of Cassia occidentalis significantly ameliorates and protects potassium bromate induced hepatotoxicity and nephrotoxicity in rats. The extracts may be protecting and ameliorating kidney related health challenges posed due to effect of process foods and toxicity of preservatives.

\section{REFERENCES}

[1]. Al-Snafi A.E (2015). The therapeutic importance of Cassia occidentalis - An overview.Indian J Pharm SciRes; 5:158 -71.

[2]. Ammon HP, Anazodo MI, Safayhi H, Dhawan BN, Srimal RC (1992). Curcumin: A potent inhibitor of leukotriene B4 formation in rat peritoneal polymorphonuclear neutrophils (PMNL). Planta Med;58:226.

[3]. Ammon HP, Wahl MA (1991). Pharmacology of Curcuma longa. Planta Medcal journal ;57:1-7.

[4]. Clement Opoku-Okrah, Benjamin KojoSafoAcquah, Elliot Eli Dogbe (2015). Changes in potassium and sodium concentrations in stored blood.The Pan African Medical Journal.20:236.

[5]. Cohly H. H, Taylor A, Angel M. F (1998), Salahudeen A. K. Effect of turmeric, turmerin and curcumin on H2O2-induced renal epithelial (LLCPK1) cell injury. Free RadicBiolMed. ;24:49-54.

[6]. Drew A. (2000), Drug discovery today and tomorrow. Drug Discovery Today 5 (1), 2-4.

[7]. Farombi E, Ekor M. (2006). Curcuminalmeliorate gentamicin induced renal oxidative damage in rats. Food Chem. Toxicol., 44(9): 1443 -1448.

[8]. Farombi, E.O., Alabi ,M.C.andAlkuru,T.O.(2000).Kolaviron modulates cellular redox status and impairment of membrane protein activities induced by potassium bromate (KBrO3) in rats. Pharmaco. Res.45, 63-68.

[9]. Fujie, M., Oikawa, K., Saito, H., Fukuhoro,C., Onosaka,S.and Tanaka, K. (1984). Metabolism of potassium bromate in rats.In vivo studies.Chemosphere. 13, 1207-1212.

[10]. Gaind, K.N., R.D. Budhiraja and R.D. Kaul (1966). Antibiotic activity of Cassia occidentalis L. Indian J. Pharm., 28: 248-250.

[11]. Govindarajan V. S (1980). Turmeric-chemistry, technology, and quality.Crit Rev Food SciNutr.;12:199-301.

[12]. Hamid Nasri, NajmehSahinfard, MortazaRafieian, Samira Rafieian, Maryam Shirzad, Mahmoud Rafieian-kopaei (2014) Turmeric: A spice with multifunctional medicinal properties.JHerbMedPharmacol.; 3(1): 5-8.

[13]. Harper HA, Rodwell VW, Mayes PA.(1979).Metabolism of carbohydrates and lipid metabolism. In: "Review of Practical a review of preclinical and clinical research. Altern Med Rev;14(2):141-153.
[14]. Haselwood, E.L. and G.G. Motter, (1966). Handbook of Hawaiian Weeds. Hawaiian Sugar Planters' Association, Honolulu, HI., USA., Pages: 479.

[15]. Henty, E.E., G.H. Pritchard and F. Owner, (1975). Weeds of New Guinea and their Control. 2nd Edn., Division of Botany, Dept. of Forests, Papua New Guinea, Pages: 180.

[16]. Ikhajiangbe H. I.N, Ezejindu D.N and Akingboye A.J. (2014) .The effect of methanolic extract of Portulacaoleraceaon potassium bromate induced nephrotoxicity in adult wistar rats.International Journal of Medicine and Medical Science Research Vol. 2(2), pp. 019-023

[17]. Isah R.T, Mohammed M.O, Muhammad A.T, Sahabi S.M, Umar Z.U, Mahmud R.I, Abubakar U (2018) Effects of Aqueous Leaf Extracts of Senna occidentalis on Rat Kidney.Afr. J. Biomed. Res. Vol. 21, 225- 230

[18]. Joe. B, Vijay Kumar. M and B. R. Lokesh.B.R (2004), "Biological properties of curcumin -cellular and molecular mechanisms of action," Critical Reviews in Food Science and Nutrition, vol. 44, no. 2, pp. $97-$ 111.

[19]. Kakehashi A, Wei M, Fukushima S, Wanibuchi H (2013). Oxidative stress in the carcinogenicity of chemical carcinogens. Cancers (Basel);5:1332-1354

[20]. Kim KJ, Yu HH, Cha JD, Seo SJ, Choi NY, You YO (2005).Antibacterial activity of Curcuma longa L. against methicillin-resistant Staphylococcus aureus. Phytother ;19(7):599-604.

[21]. Kohli K, Ali J, Ansari M, Raheman Z (2005). Curcumin: a natural anti-inflammatory agent. Indian $\mathrm{J}$ Pharmacol.;37(3):141-147.

[22]. Kopple JD, Ding H, Letoha A, Ivanyi B, Qing DP, Dux L( 2002). L-carnitine ameliorates gentamicin induced renal injury in rats. Nephrol. Dial. Transplant., 17: 2122-2131.

[23]. Kutom, A., Baziliski, N.G., Magana, L., Dunea, G. (1990). Bromate intoxication: Hairdresser's anuria. Am. J. Kidney Dis.15, 84-85

[24]. Long, R.W. and O. Lakela, ( 1976). A Flora of Tropical Florida.Banyon Books, Miami, Florida, Pages: 962.

[25]. Mahdi Thuawaini M, MawahibGasim Al-Farhaan B, Karima Abbas F (2019),hepatoprotective and nephroprotective effects of the aqueous extract of Turmeric (Curcuma longa) in rifampicin and isoniazid-induced hepatotoxicity and nephrotoxicity in rats. Asian journal of pharmaceutical and clinical researchvol 12:3.

[26]. Maizura M, Aminah A, Wan Aida W(2011). Total phenolic content and antioxidant activity of kesum (Polygonum minus), ginger (Zingiberofficinale) and turmeric (Curcuma longa) extract. Int Food Res J.1;18:526-531.

[27]. Miriyala S, Panchatcharam M, Rengarajulu P (2007). Cardioprotective effects of curcumin. The molecular targets and therapeutic uses of curcumin in health and disease.;595:359-377. 
[28]. Nnama T.N, Okafor E.C, OkaforM.C,Asebioyo S.J, Ozumba O.G and Ikpeze S.C (2019). Curative and protective efficacy of cassio occidentalis ethanolic leaf extract on carbon tetrachloride induced liver damage of female albino wistar rats.EC Clinical and Experimental anatomy 2.5;212-219.

[29]. NwaehujorChinaka O., Ode Okwoche J. and OkoyeDozie N., (2011). The Hepatoprotective Effect of Senna occidentalis Methanol Leaf Extract Against Acetaminophen-induced Hepatic Damage in Rats. Journal of Pharmacology and Toxicology, 6: 637-646.

[30]. Okolie, N.P.andIkewuchi, J.C. (2004). Cataractogenic potential of bromate-mediated oxidative stress in rabbits.Journal of Medical Scences.4, 158-163.

[31]. Oloyede OB, Sunmonu TO (2009). Potassium bromate content of selected bread samples in Ilorin, central nigeria and its effect on some enzymes of rat liver and kidney. Food Chem Toxicol;47:2067-2070.

[32]. Panchatcharam M, Miriyala S, Gayathri V.S, Suguna L (2006). Curcumin improves wound healing by modulating collagen and decreasing reactive oxygen species. Mol Cell Biochem.;290(1):87-96.

[33]. Pedraza-Chaverrí J, Maldonado PD, Medina-Campos ON, Olivares-Corichi IM, Granados-Silvestre MA, Hernández-Pando R, Ibarra-Rubio ME (2000). Garlic ameliorates gentamicin nephrotoxicity: relation to antioxidant enzymes. 2000. Free Radical Bio. Med., 29(7): 602-611.

[34]. Phansawan B, Poungbangpho S (2007). Antioxidant capacities of Puerariamirifica, Stevia rebaudianaBertoni, Curcuma longa Linn.,Andrographispaniculata (Burm. f.) Nees.and Cassia alata Linn.for the development of dietary supplement. KasetsartJ. ;41(3):407-413.

[35]. Rehab Omer AbdElhalim (2006) Biochemical Effect of Potassium Bromate on Wistar Albino Rats. $J$. Cancer Res. Clin. Oncol. 119, 463-469.

[36]. Reyes N, Gadsby DC (2006). Ion permeation through the $\mathrm{Na}+\mathrm{K}+-\mathrm{ATPase}$. Nature International weekly journal of science; 443:470-4.

[37]. Sahgal G, Ramanathan S, Sasidhara S, Mordi M, Ismail S, Mansor SM (2010). 'Brine shrimp lethality and acute oral toxicity studies on Swieteniamahagoni (Linn.) Jacq. Seed methanolic extract'. Phcog Res;2(4):215-20.

[38]. Silva, M.G.; Aragao, T.P.; Vasconcelos, C.F.; Ferreira, P.A.; Andrade, B.A.; Costa, I.M.; Costasilva, J.H.; Wanderley, A.G.; Lafayette, S.S. (2011). Acute and sub-acute toxicity of Cassia occidentalis L. stem and leaf in Wistar rats. Journal of Ethnopharmacology, 136(2): 341-346.

[39]. Timm, C.D. and F. Riet-Correa, (1997). Plants toxic to pigs.Ciencia Rural, 27: 521-528

[40]. Tirkey V, Kaur G, Vij G, Chopra K. (2005). Curcumin a diferuloylnethare, attenuates cyclosporine induced renal dysfunction and oxidative stress in rat kidneys. BMC Pharmacol., 5: 189-196.
[41]. Usha K, Mary Kasturi G, and Hemalatha P (2007). Hepatoprotective effect of Hygrophilaspinosa and Cassia occidentalis on carbon tetrachloride induced liver damage in experimental rats. Indian Journal of ClinicalBiochem.; 22(2): 132-135.

[42]. Venkatesan, N (2000). "Pulmonary protective effects of curcumin against paraquat toxicity," Life Sciences, vol. 66, no. 2, pp. 21-82

[43]. Watanabe, S., Tajima,Y. Yamaguchi,T. and Fukui, T. (2004). Potassium bromate induced hyper-uricemia stimulates acute kidney damage and oxidative stress .J.Health Sci. 50, 647-653.

[44]. World health organization (2003).Importance Natural medicinal products. Accessed $4^{\text {th }}$ may 2020.

[45]. www.kent.co.in/blog/the-harmful-effects-ofpreservatives-on-your-health/accessed , $3^{\text {rd }}$ July, 2019

[46]. Yadav J.P, Arya V, Yadav S, Panghal M, Kumar S, Dankar S; (2009). Cassia occidentalis.A review on its ethnobotany, phytochemical and pharmacological process.Fitoterapia. 10:1016 